\title{
A Single-legged Robot Inspired by the Jumping Mechanism of Click Beetles and Its Hopping Dynamics Analysis
}

\author{
Gang Chen ${ }^{12^{*}}$, Jiajun $\mathrm{Tu}^{1}$, Xiaocong $\mathrm{Ti}^{1}$, Huosheng $\mathrm{Hu}^{2}$ \\ 1. Faculty of Mechanical Engineering and Automation, Zhejiang Sci-Tech University, Hangzhou 310018, China \\ 2. School of Computer Science and Electronic Engineering, University of Essex, Colchester CO4 3SQ, UK
}

\begin{abstract}
The click beetle can jump up with a hinge when it is on the dorsal side. This jumping mechanism is simple and suitable as an inspiration for designing a simple, small, and reliable hopping robot. We report a single-legged robot inspired by the jumping mechanism of click beetles. It is $85 \mathrm{~mm}$ high, $60 \mathrm{~mm}$ long, and $41 \mathrm{~mm}$ wide, and weighs about $49 \mathrm{~g}$. The robot has good hopping performance that the hopping height is about 4 times -4.3 times of its body height. It is capable for rescue missions that require to enter enclosed spaces through cracks and narrow channels. In addition, hopping dynamics of the robot is important to understand its jumping mechanism and improve the robot's hopping performance. But existing dynamic study does not complete the analysis including all stages in the hopping which are pre-hopping, take-off, and air-flying. We propose the decomposition method to study dynamics of the three stages separately, and synthesize them with related parameters. The dynamic synthesis of multi-motion states in a hopping cycle of the single-legged hopping robot is implemented. The hopping performance and dynamic synthesis theory of the robot are verified by simulations and experiments. Our study helps lay the foundation for design and hopping control of simple hopping robot systems.
\end{abstract}

Keywords: single-legged hopping robots, hopping cycle, dynamics synthesis, multi-motion states, jumping mechanism, imitation of click beetles

Copyright (c) The author(s) 2020.

\section{Introduction}

Legged robots can access the complex terrain environments that cannot be accessed by wheeled mobile robots $^{[1,2]}$. In general, a legged hopping robot has a simple, small, and reliable mechanical structure and good dynamic acceleration performance. It can play an important role on uneven terrains and handle obstacles $^{[3,4]}$. Moreover, the legged hopping robots can walk on gullies, rocks, and other obstacles, and are extremely suitable to search and rescue operations ${ }^{[5]}$ and lunar lander ${ }^{[6,7]}$. In recent years, many researchers have studied the legged hopping robots ${ }^{[8-10]}$.

Link mechanism including the open and parallel chain was applied for jumping design. Vu et al. studied a two-segmented leg with a knee and hip joints that implemented hopping movement using the variable stiffness mechanism ${ }^{[11]}$. Zhang et al. built a two-wheeled hopping robot with a four-bar linkage ${ }^{[12]}$. Its gear train has high transmission efficiency. The four-bar linkages are light weight and have high jumping efficiency.
Kenneally et al. proposed the design principles for a family of direct-drive legged robots, in which the structural design of the leg-type hopping robot was stu$\operatorname{died}^{[13]}$. Jung et al. used the symmetric five-bar structure to design a jumping leg for the integrated jumping-crawling robot ${ }^{[14]}$. Haldane et al. presented a robotic galago which utilized a power-modulating strategy to obtain higher peak power ${ }^{[15]}$. Kovač et al. described a spherical system which was able to jump, upright itself after landing and jump again based on a jumping robot $^{[16]}$. Plecnik et al. designed a novel jumping robot, Salto. It is an eight-bar linkage with a stroke greater than half the robot's total height ${ }^{[17,18]}$. These linkage hopping robots presented good jumping performance, but also have some problems: (1) The structures are complex, which are formed with several links. (2) The legs should be supported to stand before jumping. Thus, they cannot jump by themselves.

Natural jumping creatures have good jumping ability to overcome large obstacles. Some hopping legs were inspired by them, such as the kangaroo, locust, and

\footnotetext{
*Corresponding author: Gang Chen

E-mail: gchen@zstu.edu.cn
} 
frog. Graichen et al. presented a bionic kangaroo robot. Its knee and ankle joints are connected via coupled kinematics, and bidirectional pneumatic actuators are mounted at the lower legs ${ }^{[19]}$. Some jumping robots were designed with the inspirations of locusts. Scarfogliero et $a l$. created a long-jumping "Grillo" mini robot with the tiny motors that drives the eccentric $\mathrm{cam}^{[20]}$. It was cooperated with the escapement compression release torsion spring to jump ${ }^{[21]}$. Beck et al. proposed a locust-inspired miniature jumping robot $^{[22]}$. The basic mechanism is similar to that of the semilunar process in the hind legs of the locust, and two torsion springs joining a pair of legs connected through a revolute joint to an electric motor ${ }^{[23]}$. Nguyen and Park presented a locust-inspired jumping mechanism prototype that used springs, wire, reduction gears, and a motor as the actuation components ${ }^{[24]}$. Wang et al. studied a mechanical model of jumping robot based on the biological mechanism analysis of frog ${ }^{[25]}$. The robot's leg was designed with a 4-bar spring/linkage mechanism model. Reddy et al. presented a frog-inspired robot. It was created using a four-bar spring/linkage mechanism integrated with spool winding and ratchet release mechanism $^{[26]}$. The hopping robots inspired by natural jumping creatures achieved good jumping characteristics, but still present some problems: (1) Natural jumping creatures usually jump with rear legs which have no other use, and their size accounts for a large proportion of the overall body. Thus, the dimensions of the inspired jumping legs are relatively large, and small and compact structures are differently obtained. (2) Rear legs of the natural jumping creatures usually need other legs to help for jumping. Therefore, the inspired jumping legs cannot succeed to jump independently.

A simple, small, compact, and independent hopping robot is important for rescue missions that require it to enter enclosed spaces, such as collapsed buildings and caves, through cracks and narrow channels ${ }^{[27]}$. The existing jumping robots cannot fit the above demands.

Click beetles (Elateridae sp, see Fig. 1a) are characterized by the unusual jumping ability owing to its unique structures ${ }^{[28]}$. Its hinge part (see Fig. 1b) connects the front body and the rear body ${ }^{[29]}$. The front body contains the head and prothorax, and the rear body includes the mesothorax, metathorax and abdomen ${ }^{[30]}$.
When the click beetle is on its dorsal side, it arches its back around the hinge and the middle of the body is raised slightly from ground. This process stores energy in enlarged muscles in their prothorax, cuticle, tendons and other biomechanical springs for jumping. Then muscles abruptly releases energy and the robot jumps up.

Inspired by the jumping mechanism of click beetles, we design a single-legged hopping robot. It is simple, small, compact, and independent enough to complete the rescue missions. The hopping process of the natural jumping creatures and robots can be divided into three stages: pre-jumping, take-off, and air-flying stage. The dynamics of the hopping process is important to understand its jumping mechanism and improve the robot's hopping performance. Some studies have been done on this subject.

Chen et al. used the dynamic equation to study the landing state of a hopping robot, the influence of the robot body posture and each leg on the stability of the cushioning performance ${ }^{[32]}$. Zhao et al. conducted the research on a single-motor-actuated miniature steerable jumping robot, including a dynamic analysis of the robot's take-off phase through momentum conservation and kinetic energy theorem to obtain the optimal mechanism size of the robot ${ }^{[33]}$. This method has good versatility and can be used for other robot design analysis. Zhang et al. proposed a jump model with good dynamic performance and analyzed the discrete parameters of the trailing trajectory of the hind legs of the locusts during the take-off process to optimize the structural parameters ${ }^{[34]}$. Li et al. analyzed the dynamics of the robot's take-off phase through the study of the biological characteristics of insects and proposed the use of contact force of the jump acceleration phase to the ground to optimize the robot jump ${ }^{[35]}$. The research in Refs. $[24,36]$ was mainly focused on the dynamic analysis of air flying and few kinetic analyses for pre jumping and taking off.

The existing dynamic analysis focuses on one stage of the hopping process. While the three stages are all significant for the robot's hopping. Therefore, we analyse the dynamics of the multi-motion states in its entire hopping cycle and implement the dynamics synthesis of the click beetle inspired robot which can lay a foundation for the research of hopping robot dynamics. 


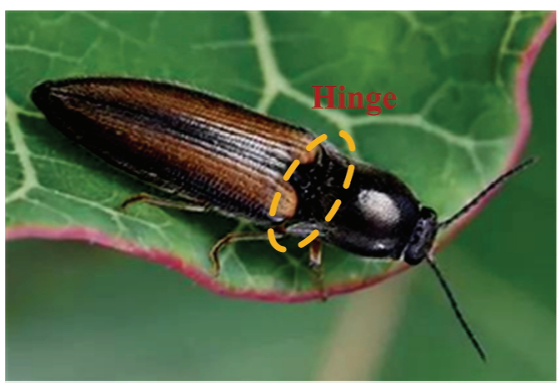

(a)

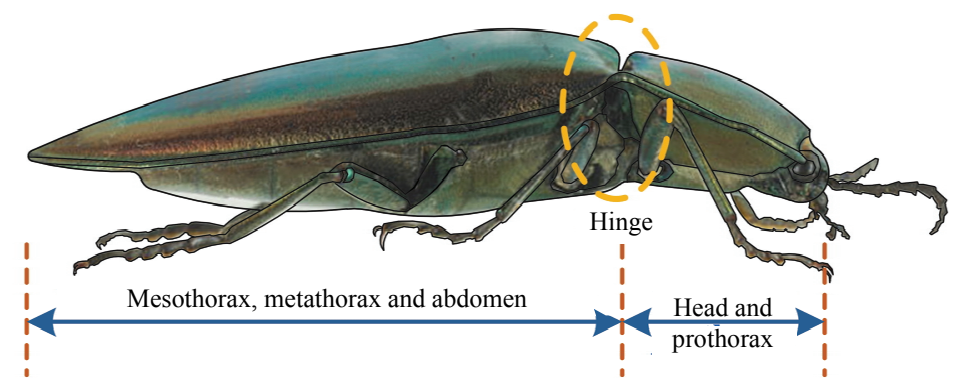

(b)

Fig. 1 Click beetle and its structure. (a) A click beetle (Elateridae sp) on a leaf ${ }^{[31]}$; (b) sturcture of a click beetle which contains the head and prothorax and the mesothorax, metathorax and abdomen and they are connected by a hinge ${ }^{[30]}$.

In the following, we would like to present a simple, small, compact, and independent single-legged hopping robot with the inspiration of the jumping mechanism of click beetles. Afterwards, the dynamics of the multi-motion states of the robot in its entire hopping cycle is analyzed. Next, the fabrication method of the single-legged hopping robot and the simulation and experiment procedures are described. Then, simulation and experiment results of the hopping process of the robot are presented to demonstrate the feasibility and performance of the developed single-legged hopping robot and verify the validity of the dynamic model. Finally, the single-legged hopping robot, dynamics of the robot, and future work are discussed and concluded.

\section{Materials and methods}

\subsection{Design of a single-legged hopping robot inspired by the jumping mechanism}

The hinge of a click beetle is the important part for jumping, and its anatomy is depicted in Fig. $2 \mathrm{a}^{[37]}$. The hinge has a peg-hold and a mesothermal lip and some muscles distribute around. The jumping process of a click beetle can be divided into three stages: pre-jumping stage, take-off stage, and air-flying stage, in which the hinge and muscles performs in different states. Fig. $2 \mathrm{~b}$ describes the jumping process. In the pre-jumping stage, the peg-hold moves along the mesosternal lip and stops when its friction plate drops into a semi-spherical ledge of the mesosternal lip. The front and rear bodies of a click beetle move around the hinge while its middle is raised. The elastic energy is stored with powerful muscles ${ }^{[38]}$. When the energy is released, the take-off stage starts. The peg-hold moves back over the mesosternal lip and into the cavity and the front and rear bodies move around the hinge in the opposite direction compared with the pre-jump stage. The body hits the ground and gets the force to launch itself. When the click beetle leaves the ground, the air-flying stage begins. The click beetle flies in the air and rolls, and its gravity centre passes a curve trajectory in the air. Finally, it lands on its feet or still on its dorsal side randomly.

It is clear that two aspects play the most important role in the jumping process of a click beetle. One is the hinge part which is the basic structure and adjusts the body morphology during the jumping. The other one is the muscle which stores and abruptly releases energy to provide the power for jumping. As shown in Fig. 3a, when front and rear bodies of the click beetle contact the ground in the pre-jumping stage, they can be equivalent to two linkages which are connected by a hinge. Inspired by the jumping mechanism of click beetles, a single-legged hopping robot is designed as shown in Figs. $3 \mathrm{~b}$ and $3 \mathrm{c}$. The parameters of the robot are shown in Table A1. It is $85 \mathrm{~mm}$ tall and $60 \mathrm{~mm}$ long, and weighs $49 \mathrm{~g}$. The robot includes the main and lower legs, a hinge, a partial gear set, and energy storage springs. The deformation of the torsion spring accumulates and suddenly releases the energy to provide the power for hopping. As shown in Fig. 3c, the two ends of the spring are hooked and applied to the main leg and the lower leg. The torsion springs are used as an energy storage device with power amplification and high triggering efficiency. The main leg and lower leg are made from high-performance nylon material and have through holes for mounting the hinge shaft.

The sector gear is mounted coaxially with the lower 


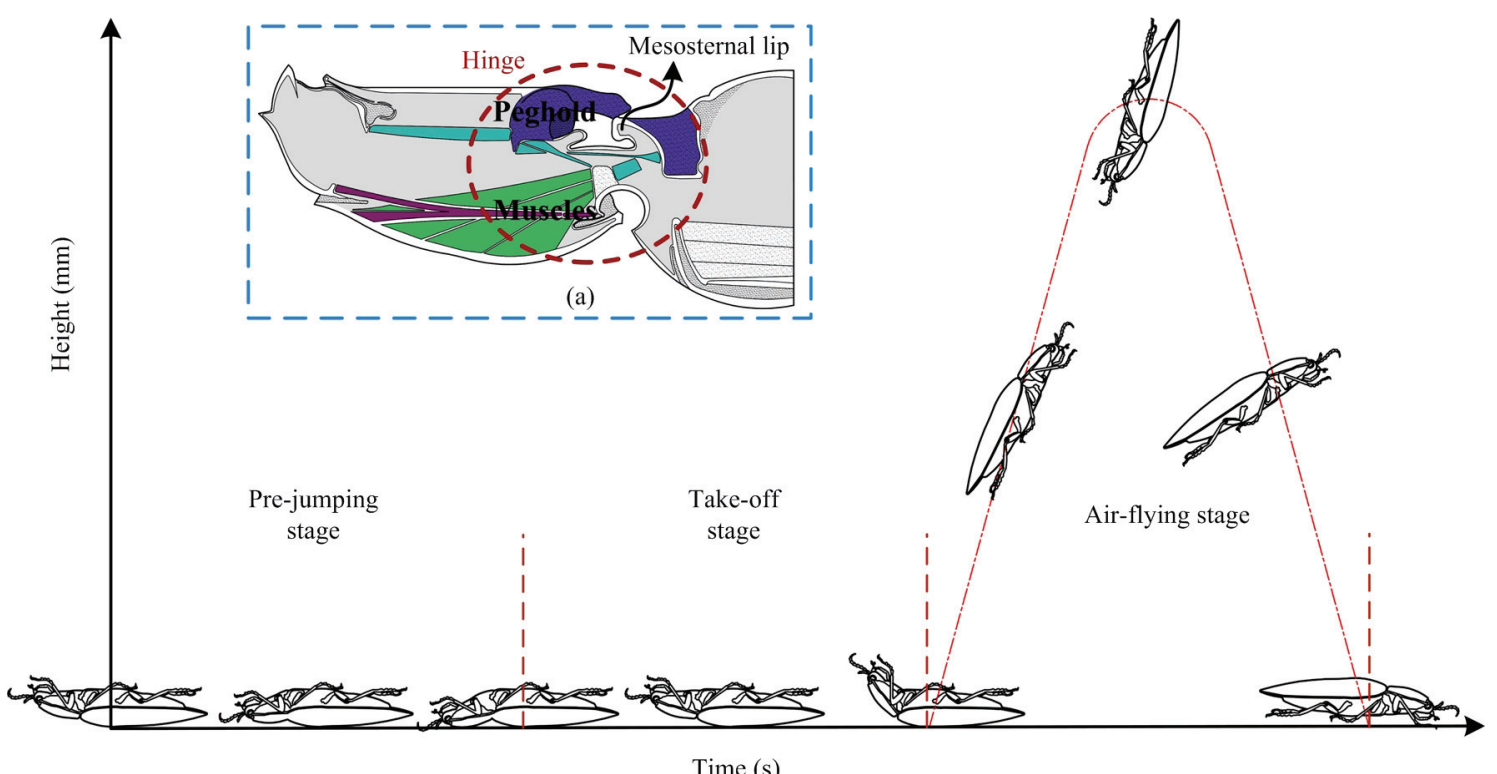

(b)

Fig. 2 Jumping process of a click beetle ${ }^{[30,37]}$. (a) Anatomy of the hinge of a click beetle. The hinge has a peg-hold, a mesothermal lip and some muscles, which coordinate and implement the jumping motion ${ }^{[39]}$. (b) Three stages in the jumping process of a click beetle, which are pre-jumping, take-off, and air-flying stage.

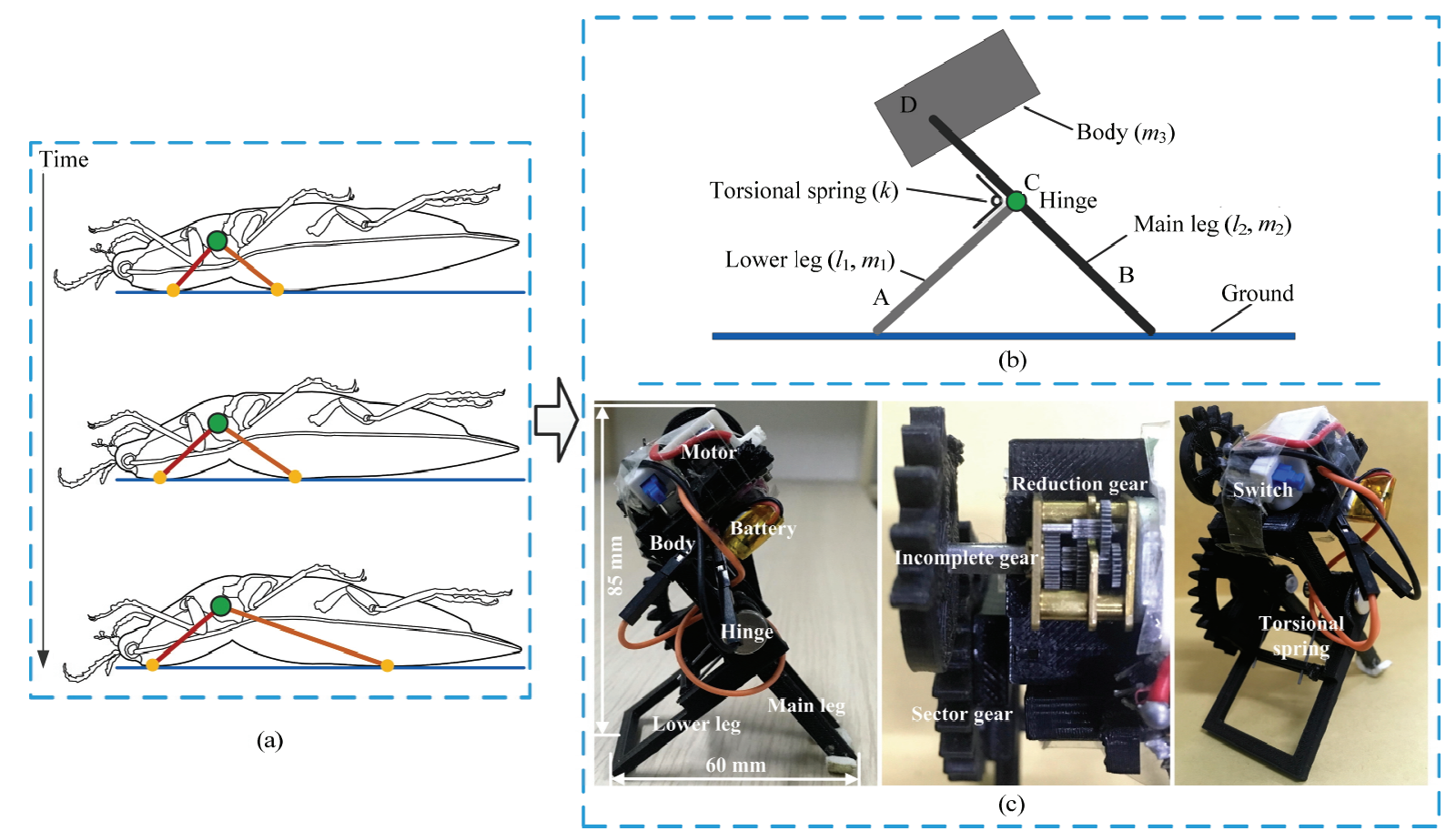

Fig. 3 A single-legged hopping robot inspired by the jumping mechanism. (a) Morphology of a click beetle when front and rear bodies contact ground in the pre-jumping stage. The click beetle can be considered as two linkages connected through a hinge. (b) Simplified schematic of a single-legged hopping robot inspired by the jumping mechanism, which has two legs and rotate round a hinge. (c) Three photos of the single-legged hopping robot platform taken from different directions. The robot is $85 \mathrm{~mm}$ tall and $60 \mathrm{~mm}$ long.

hinge shaft and is fixed to the lower leg. The body is rigidly connected to the main leg, which is equipped with a battery, reduction gears, and a motor. The output shaft of the motor relates to a reduction gear to increase the output torque. The incomplete gear is driven by the motor and has the characteristics of simple structure and 
high transmission efficiency. The sector gear connected to the lower leg is driven by the incomplete gear. The lower joint torsion spring is compressed by rotating clockwise around the hinge. The joint angle between the two legs reduces and causes the entire robot to squat downward with the main leg sliding on the ground, and that consequently reduces the height of its centre of gravity. When the incomplete gear rotates to the empty-tooth part, it disengages from the sector gear and the torsion spring releases the elastic potential energy instantaneously. The torque is converted into the leg-to-ground force through the main and lower legs, and the ground provides a vertical upward reaction force and a horizontal forward friction. Great friction can be obtained with the pointed foot of the front leg for the light robot. When the gears disengage until the robot leaves the ground, the reaction force of the ground provides a large starting kinetic energy for its horizontal and vertical motion. During the robot is launched to the air, the spring relaxes, each part is on its initial state, the robot rolls, and its gravity centre flies through a curve. Finally, the robot lands on the ground and completes one hopping. Thus, multi-motion states exist in one cycle hopping process of the robot.

One cycle hopping process can be divided into three stages: the pre-hopping, take-off, and air-flying stage, as shown in Fig. 4. In the pre-hopping stage, the motor drives the incomplete gear which rotates on the sector gear. The torsion spring installed on the hinge is compressed and the height of the gravity centre of the robot decreases continuously. When the teeth of the incomplete gear and sector gear separate with each other and the torsion spring releases, the take-off stage starts, which ends at the moment when the robot leaves the ground. During this stage, each part of the robot recovers to its initial state and the height of its gravity centre increases continuously. Once the robot leaves the ground, the air-flying stage begins. The robot flies and rolls through a trajectory in the air until the robot lands on ground. When the robot lands on the ground, it will be impacted. The main and lower legs, incomplete gear, and sector gear were manufactured through $3 \mathrm{D}$ printing with the high-performance nylon, and the weight of the robot is light. Thus, they are affected little by the collisions. But the impact will destroy the motor and battery of the robot. We use the sponge to wrap them around to reduce the influence. The sponge is light and has good damping performance, so it is suitable for protecting the motor and battery from impact.

\subsection{Dynamic analysis of the multi-motion states of the single-legged hopping robot}

\subsubsection{Pre-hopping stage}

In this stage, the motor rotates and meshes with the sector gear through the incomplete gear. The main leg is twisted clockwise and the height of the gravity centre of the robot is reduced, and the spring is compressed, as shown in Fig. 5. Consequently, the gravitational potential energy decreases continuously, the mechanical energy is converted into the elastic potential energy, and the energy stored in the torsion spring increases continuously.

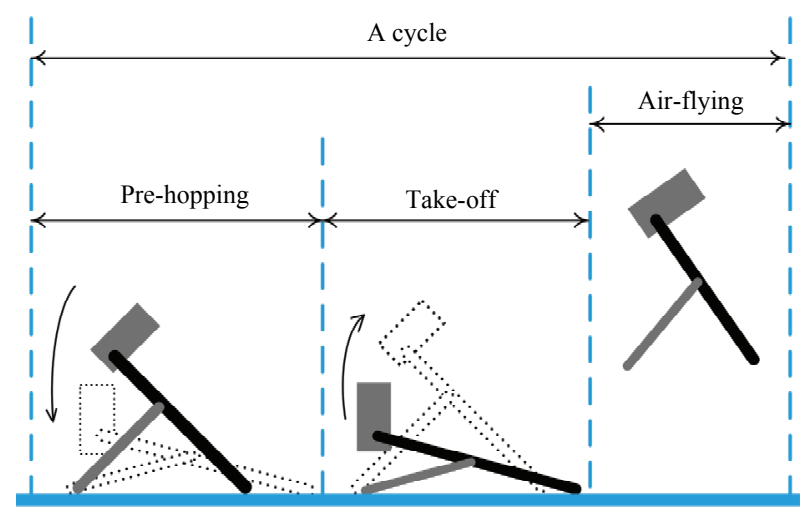

Fig. 4 A simplified schematic of hopping process of the robot. There are three stages in a hopping cycle, which are pre-hopping, take-off, and air-flying stage.

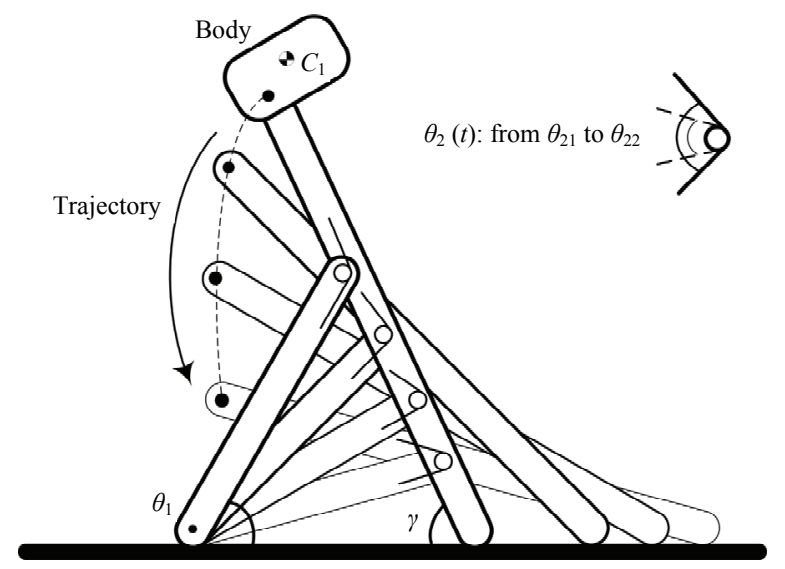

Fig. 5 A simplified schematic of the pre-hopping stage. Gravity centre of the robot goes down and the torsion spring is compressed and the spring energy increases and prepares for the take-off stage. 
About the lower leg, the required inertia force and moment of inertia are computed below:

$$
\begin{aligned}
{ }^{1} \boldsymbol{F}_{1}=m_{1}{ }_{1} \dot{\boldsymbol{v}}_{c 1}=\left[\begin{array}{c}
-m_{1} l_{1} \dot{\theta}_{1}^{2}+m_{1} g \sin \theta_{1} \\
m_{1} l_{1} \ddot{\theta}_{1}+m_{1} g \cos \theta_{1} \\
0
\end{array}\right], \\
{ }^{1} \boldsymbol{N}_{1}=\left[\begin{array}{l}
0 \\
0 \\
0
\end{array}\right] .
\end{aligned}
$$

About the main leg, the required inertia force and moment of inertia are computed as Eq. (3).

$$
{ }^{2} \boldsymbol{N}_{2}=\left[\begin{array}{l}
0 \\
0 \\
0
\end{array}\right] .
$$

The active torque generated by rotation $\tau$ is calculated as Eq. (5).

The component of ${ }^{2} \boldsymbol{n}_{2}$ is integrated in the $Z$ direction and the joint torque is obtained by Eq. (6).

$$
\begin{aligned}
\tau(t)= & m_{2} l_{22}^{2}\left(\ddot{\theta}_{1}+\ddot{\theta}_{2}\right)+m_{2} l_{1} l_{22} \dot{\theta}_{1}^{2} \sin \theta_{2} \\
& +m_{2} l_{1} l_{22} \ddot{\theta}_{1} \cos \theta_{2}+m_{2} g l_{22} \cos \left(\theta_{1}+\theta_{2}\right)+n_{s},
\end{aligned}
$$

where $n_{s}$ is the spring torque.

The motor input has a constant angular velocity $\omega$ as:

$$
\begin{gathered}
\theta_{2}=\left(\theta_{21}+\frac{a}{b} \omega \cdot t\right), \\
\ddot{\theta}_{1}=\ddot{\theta}_{2}=0 .
\end{gathered}
$$

Therefore, Eq. (6) becomes:

$$
\begin{aligned}
\tau= & m_{2} l_{1} l_{22}\left(1.2 \frac{a}{b} \omega \cdot t\right)^{2} \sin \left(\theta_{21}+\frac{a}{b} \omega \cdot t\right) \\
& +m_{2} g l_{22} \cos \left[0.2\left(\theta_{21}+\frac{a}{b} \omega \cdot t\right)\right]+k \frac{a}{b} \omega t,
\end{aligned}
$$

where $\theta_{21}$ is the initial angle.

The torque of the joint angle increases with time. At different input speeds, the larger velocity is and the shorter the required torque is. The torque obtained at the end of this stage is used as the driving torque in the subsequent stage to enable the robot to obtain the hopping energy within a short time. We analyse the bounce speed of the robot when the gear is disengaged in the take-off stage.

\subsubsection{Take-off stage}

As can be seen in Fig. 6, the torsion spring stores a large amount of elastic potential energy during the pre-hopping stage. When the incomplete gear is about to be disengaged, the torsion spring is twisted to the limit position and the centre of gravity of the robot is reduced by $1 / 2$ of the original height. At this time, the elastic spring energy stored in the torsion spring is the largest. The previous resistance torque becomes the output torque $\tau^{\prime}(t)$. When the gear is disengaged, the bouncing robot recovers rapidly under the action of the torsion spring force. During the period, the elastic force overcomes the gravity and the friction at the joint to perform the task, which is converted into the kinetic energy of the robot.

Newton-Eulerian iterative dynamic equation of the lower leg at the take-off stage is:

$$
\begin{aligned}
& { }^{2} \boldsymbol{F}_{2}=m_{2}{ }^{2} \dot{\boldsymbol{v}}_{c 2} \\
& =\left[\begin{array}{c}
-m_{2} l_{22}\left(\dot{\theta}_{1}+\dot{\theta}_{2}\right)^{2}-m_{2} l_{1} \dot{\theta}_{1}^{2} \cos \theta_{2}-m_{2} l_{1} \ddot{\theta}_{1} \sin \theta_{2}+m_{2} g \sin \left(\theta_{1}+\theta_{2}\right) \\
m_{2} l_{22}\left(\ddot{\theta}_{1}+\ddot{\theta}_{2}\right)+m_{2} l_{1} \dot{\theta}_{1}^{2} \sin \theta_{2}+m_{2} l_{1} \ddot{\theta}_{1} \cos \theta_{2}+m_{2} g \cos \left(\theta_{1}+\theta_{2}\right) \\
0
\end{array}\right] . \\
& { }^{2} \boldsymbol{n}_{2}={ }^{2} \boldsymbol{N}_{2}+{ }_{3}^{2} \boldsymbol{R}^{3} \boldsymbol{n}_{3}+{ }^{2} \boldsymbol{P}_{\mathrm{c} 2} \times{ }^{2} \boldsymbol{F}_{2}+{ }^{1} \boldsymbol{P}_{2} \times{ }_{3}^{2} \boldsymbol{R}^{3} \boldsymbol{f}_{3} \\
& =\left[\begin{array}{c}
0 \\
0 \\
m_{2} l_{22}^{2}\left(\ddot{\theta}_{1}+\ddot{\theta}_{2}\right)+m_{2} l_{1} l_{22} \dot{\theta}_{1}^{2} \sin \theta_{2}+m_{2} l_{1} l_{22} \ddot{\theta}_{1} \cos \theta_{2}+m_{2} g l_{22} \cos \left(\theta_{1}+\theta_{2}\right)
\end{array}\right] .
\end{aligned}
$$




$$
{ }^{1} \boldsymbol{F}_{1}=m_{1}{ }_{1} \dot{\boldsymbol{v}}_{c 1}=\left[\begin{array}{c}
-m_{1} l_{1} \dot{\theta}_{1}^{2}+m_{1} g \sin \theta_{1} \\
m_{1} l_{1} \ddot{\theta}_{1}+m_{1} g \cos \theta_{1} \\
0
\end{array}\right] .
$$

The inertia force and moment of the main leg are computed as Eqs. (11) and (12).

The joint torque, which is the $Z$ component of ${ }^{2} \boldsymbol{n}_{2}$, is obtained as:

$$
\begin{aligned}
\tau= & m_{2} l_{22}^{2}\left(\ddot{\theta}_{1}+\ddot{\theta}_{2}\right)+m_{2} l_{1} l_{22} \dot{\theta}_{1}^{2} \sin \theta_{2} \\
& +m_{2} l_{1} l_{22} \ddot{\theta}_{1} \cos \theta_{2}+m_{2} g l_{22} \cos \left(\theta_{1}+\theta_{2}\right) .
\end{aligned}
$$

In this stage, the torsion spring provides the moment for take-off.

Thus, we have

$$
\begin{aligned}
n_{s}= & m_{2} l_{22}^{2}\left(\ddot{\theta}_{1}+\ddot{\theta}_{2}\right)+m_{2} l_{1} l_{22} \dot{\theta}_{1}^{2} \sin \theta_{2} \\
& +m_{2} l_{1} l_{22} \ddot{\theta}_{1} \cos \theta_{2}+m_{2} g l_{22} \cos \left(\theta_{1}+\theta_{2}\right) .
\end{aligned}
$$

As $n_{s}$ is linear with $\theta_{2}$, we assume:

$$
n_{s}=\mathrm{k} \cdot\left(180^{\circ}-\theta_{2}\right) \text {. }
$$

Therefore, we can get

$$
\begin{aligned}
k\left(180^{\circ}-\theta_{2}\right)= & 0.5 m_{2} l_{22}^{2} \ddot{\theta}_{2}+0.25 m_{2} l_{1} l_{22} \dot{\theta}_{1}^{2} \sin \theta_{2} \\
& -0.5 m_{2} l_{1} l_{22} \ddot{\theta}_{2} \cos \theta_{2} \\
& +m_{2} g l_{22} \cos \left(90^{\circ}+0.5 \theta_{2}\right) .
\end{aligned}
$$

In the dynamic analysis of the hopping process, the joint moment at the end of the pre-hopping stage is obtained as the initial moment at the beginning of the take-off stage. The relation between angular acceleration $\ddot{\theta}_{2}(t)$ and angle $\theta_{2}(t)$ is obtained by the Newton-Euler equation. The expression of $\theta_{2}(t)$ to time is obtained by solving the second-order differential equation. The maximum time $t_{2}$ of the take-off stage and the analytic expression of $\dot{\theta}_{2}(t)$ are obtained by differentiation.

\subsubsection{Air-flying stage}

Fig. 7 shows the air-flying stage of the robot in the hopping process. During this stage, the hopping robot can be regarded as a mass $m$. The motion equation of the hopping can be expressed by Newton's second law $F=\sum m a$, where $F$ is the total force acting on the hopping robot and $a$ is the acceleration.

In this case, the force is gravity, which is an important factor in energy loss. The instantaneous velocity of the hopping in the $x$ and $y$ directions is $\dot{x}(t)$ and $\dot{y}(t)$, respectively. The take-off angle is obtained as:

$$
\gamma=\tan ^{-1}\left(\dot{y}\left(t_{0}\right) / \dot{x}\left(t_{0}\right)\right) .
$$

When the robot leaves the ground, the speed is computed below:

$$
\begin{aligned}
& \dot{x}_{0}=v_{2} \cos \gamma, \\
& \dot{y}_{0}=v_{2} \sin \gamma,
\end{aligned}
$$

where $v_{2}=\dot{\theta}_{2} \cdot l_{22}$, the joint angular velocity $\dot{\theta}_{2}$ is obtained from the take-off stage.

Thus, the relationship among the robot flying height, distance, and time is obtained. The height is computed as:

$$
y(t)=\dot{y}_{0} t-\frac{1}{2} g t^{2} .
$$

The distance is obtained below:

$$
x(\mathrm{t})=\dot{x}_{0} t
$$

$$
\begin{aligned}
& { }^{2} \boldsymbol{F}_{2}=m_{2}{ }^{2} \dot{\boldsymbol{v}}_{c 2}=\left[\begin{array}{c}
-m_{2} l_{22}\left(\dot{\theta}_{1}+\dot{\theta}_{2}\right)^{2}-m_{2} l_{1} \dot{\theta}_{1}^{2} \cos \theta_{2}-m_{2} l_{1} \ddot{\theta}_{1} \sin \theta_{2}+m_{2} g \sin \left(\theta_{1}+\theta_{2}\right) \\
m_{2} l_{22}\left(\ddot{\theta}_{1}+\ddot{\theta}_{2}\right)+m_{2} l_{1} \dot{\theta}_{1}^{2} \sin \theta_{2}+m_{2} l_{1} \ddot{\theta}_{1} \cos \theta_{2}+m_{2} g \cos \left(\theta_{1}+\theta_{2}\right) \\
0
\end{array}\right] . \\
& { }^{2} \boldsymbol{n}_{2}={ }^{2} \boldsymbol{P}_{\mathrm{c} 2} \times{ }^{2} \boldsymbol{F}_{2} \\
& =\left[\begin{array}{c}
0 \\
0 \\
m_{2} l_{22}^{2}\left(\ddot{\theta}_{1}+\ddot{\theta}_{2}\right)+m_{2} l_{1} l_{22} \dot{\theta}_{1}^{2} \sin \theta_{2}+m_{2} l_{1} l_{22} \ddot{\theta}_{1} \cos \theta_{2}+m_{2} g l_{22} \cos \left(\theta_{1}+\theta_{2}\right)
\end{array}\right] .
\end{aligned}
$$




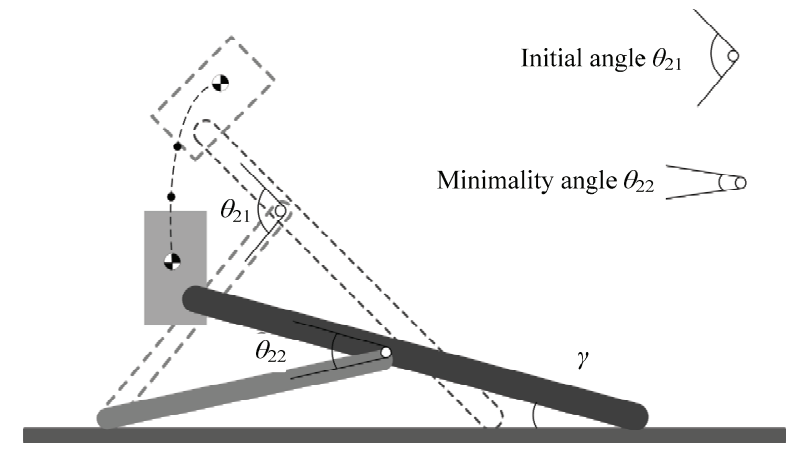

Fig. 6 A simplified schematic of the take-off stage. Gravity centre of the robot goes up and the torsion spring is released and the velocity of the robot increases and prepares for the air-flying stage.

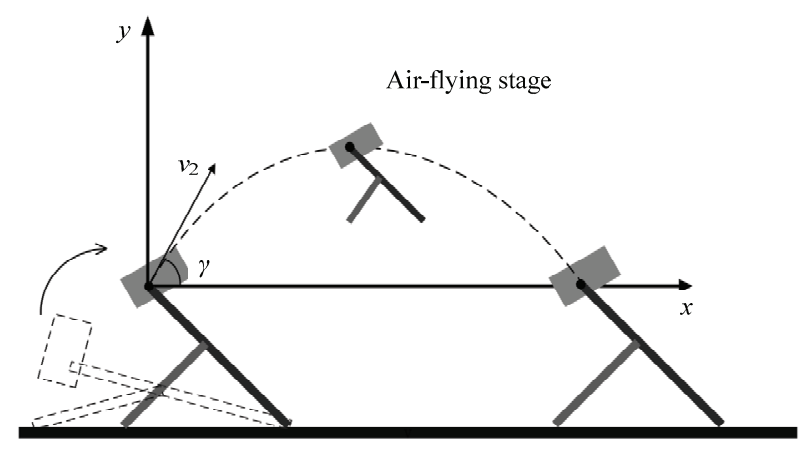

Fig. 7 A simplified schematic of the air-flying stage. The robot leaves from ground with a velocity and goes in air under the effect of gravity, finally gets back down.

\subsection{Fabrication of the robotic prototype and simu- lation and experiment procedures}

\subsubsection{Fabrication of the robotic prototype}

The main and low legs, incomplete gear, and sector gear were manufactured through $3 \mathrm{D}$ printing with the high-performance nylon: HP3DHR-PA12. A motor, contains a DC motor, a reducer was chosen, whose rated voltage is $3 \mathrm{~V}$ and rated speed is $15 \mathrm{r} \cdot \mathrm{min}^{-1}$. The system was powered by a $3.7 \mathrm{~V} 280 \mathrm{~mA}$-hour lithium polymer battery which is pasted to the main leg. The white cover was printed with resin to install the motor with the main leg. Two pins are selected according to the dimensions and as the hinge part of the robot. Two torsion springs are chosen and installed in the hinge. In order to save space and minimize the size of the robot, no control board was used. The battery was connected with the motor through a switch. When the switch is open, the motor obtains the energy from battery and starts to jump.

\subsubsection{Simulation and experiment procedures}

The simulation and experiment were conducted to verify the correctness of the proposed hopping dynamic theory and test the performance of the single-legged hopping robot inspired by the jumping mechanism of click beetles.

ADAMS software was used for simulation. The three-dimensional model of the single-legged hopping robot platform was imported into ADAMS, and the materials of the components and kinematic pairs between them were set as same as the robot platform. Rotation driving was applied on the motor, and the torsional spring load was set around the hinge part. Thus, the robot model (see Fig. 8a) was finally developed in ADAMS. It was same as the single-legged hopping robot platform. In the simulation, the angular velocity of the motor was controlled to simulate the hopping process which completed two examples with $\omega_{1}=240^{\circ} \cdot \mathrm{s}^{-1}$ and $\omega_{2}=180^{\circ} \cdot \mathrm{s}^{-1}$. The joint torque in the pre-hopping stage, angular velocity in the take-off stage, and the trajectory values in the air-flying stage were measured and recorded. The data comparing with theoretical values were used to verify the validity of the proposed hopping dynamic theory.

As shown in Fig. 8b, a high-speed camera placed perpendicular to the hopping plane was used in analyzing the performance of the single-legged hopping robot. The camera operated at 30 frames per second to capture hopping from start to end. No sensors were installed in the robot platform because of the space limitation, the joint torque joint torque in the pre-hopping stage and angular velocity in the take-off stage could not be

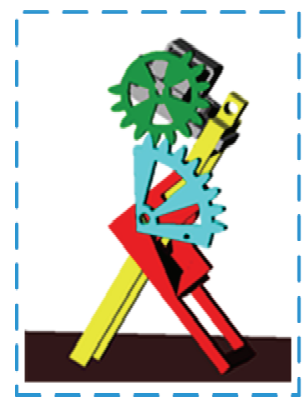

(a)

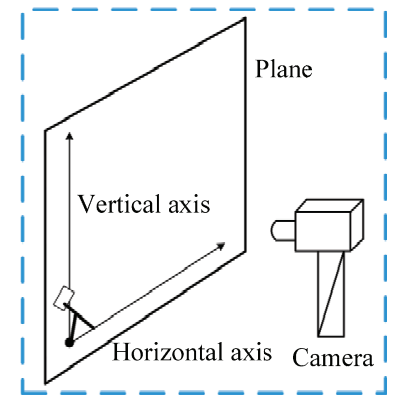

(b)
Fig. 8 Simulation and experiment platforms. (a) A single-legged hopping robot platform established in ADAMS; (b) experiment platform built with the single-legged hopping robot, a camera, and a grid board. 
obtained. From the hopping video, the hopping distance was got and used to analyse the hopping performance of the robot platform combining with the simulation and theory data. The hopping distance in the air-flying stage was related with the forward two stages, so the experiment result can also be used to verify the validity of the proposed hopping dynamic theory.

\section{Results}

The single-legged hopping robot driven with different angular velocities is simulated in ADAMS. The actuating speeds used in the simulation are $\omega_{1}=240^{\circ} \cdot \mathrm{s}^{-1}$ and $\omega_{2}=180^{\circ} \cdot \mathrm{s}^{-1}$. Fig. 9 shows the joint torque of theory and simulation results in the pre-hopping stage. The joint torque increases linearly with time in the theoretical calculation because the robot is driven at a fixed angular velocity to complete the process.

The simulation curve is fluctuated because of the force disturbances in the gear meshing, but consistent with the theoretical curve. In the pre-hopping stage, the torsion spring is twisted to the maximum, the joint torque reaches the maximum value, and the incomplete gear is disengaged from the sector gear at the next moment. In the take-off stage, the torsion spring releases in a very short time and the joint torque drops to 0 . The elastic potential energy stored in the torsion spring releases instantaneously and that will cause the torsion spring vibrate around the natural state. Thus, the joint

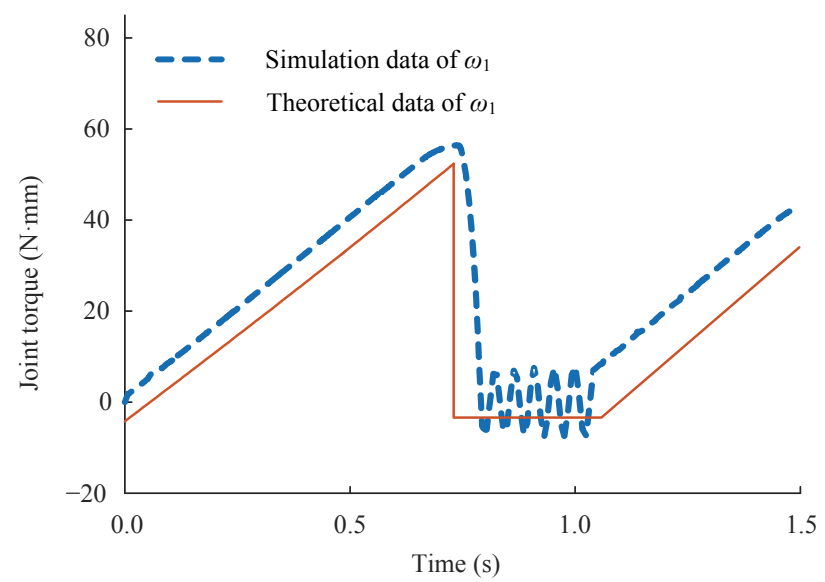

(a) torque varies around zero when the torsion spring loose in the simulation, and this aspect is not considered in the theory calculation. From the results, we can see that the simulation results are highly consistent with the theoretical calculations.

Fig. 10 shows the angular velocity of theory and simulation results in the take-off stage. At the end of this stage in simulation, the angular velocity is slightly larger than that in the theoretical calculation. The influence on the final angular velocity is not obvious. The start time of the take-off stage with the actuating speed $\omega_{1}$ is earlier than that with the actuating speed $\omega_{2} . \omega_{1}$ is greater than $\omega_{2}$. Thus, the pre-jumping process with $\omega_{1}$ is faster and the take-off stage can start earlier compared with that in $\omega_{2}$ angular velocity. The kinetic energy obtained by the robot in the take-off stage is converted into the flying velocity for the air-flying stage. In the simulation, the angular velocity varies in the take-off stage, which is caused by the torsion spring relaxation as in Fig. 9. This factor is not taken into account in the theoretical calculation.

The dynamics results of the air-flying stage are shown in Fig. 11. At the vertical height, the simulation curve rise parabolically as the theoretical curve. Although the simulated slope is similar to the theoretical curve in the horizontal direction, it does not reach the distance of the theoretical curve. It is clear that when the air-flying phase starts, a rolling phenomenon of the robot

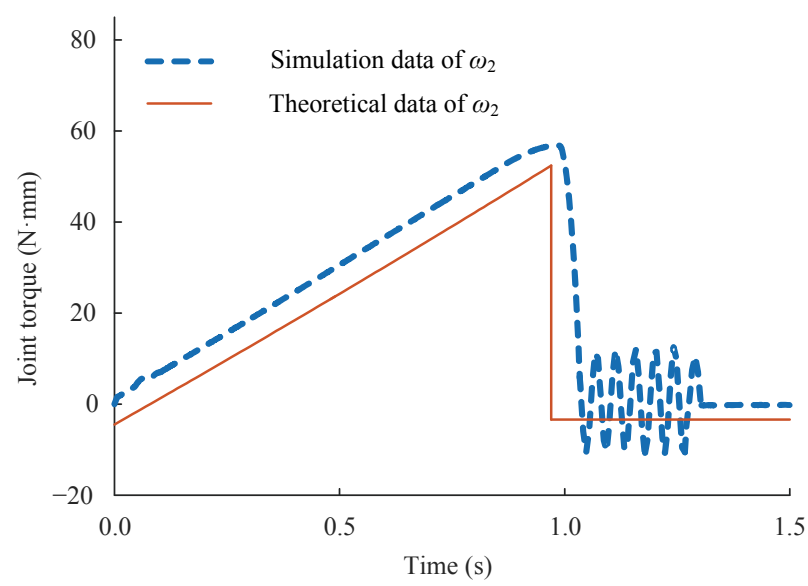

(b)

Fig. 9 Joint torques of theory and simulation results in the pre-hopping stage. (a) Joint torques in theory calculation and simulation with the actuating speed $\omega_{1}$. (b) Joint torques in theory calculation and simulation with the actuating speed $\omega_{2}$. 


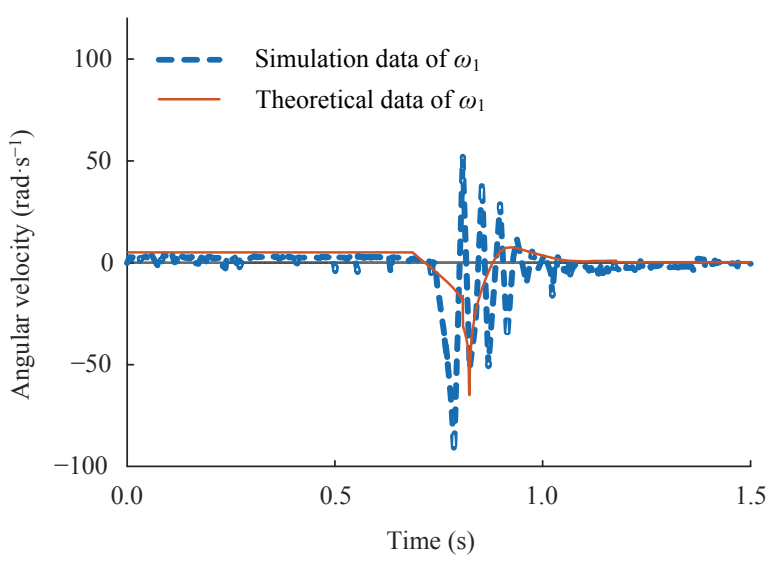

(a)

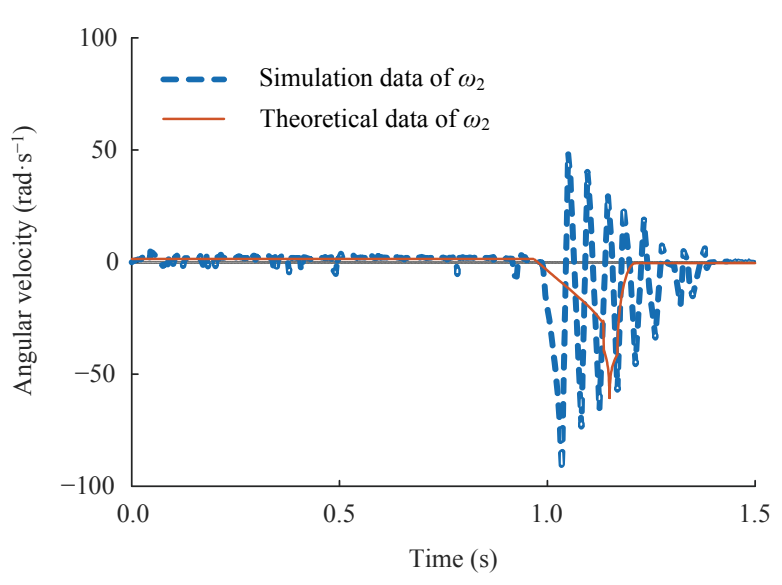

(b)

Fig. 10 Angular velocities of theory and simulation results in the take-off stage. (a) Angular velocities in theory calculation and simulation with the actuating speed $\omega_{1}$; (b) angular velocities in theory calculation and simulation with the actuating speed $\omega_{2}$. Vibrations of data in simulation were caused by the spring which was ignored in theory model.

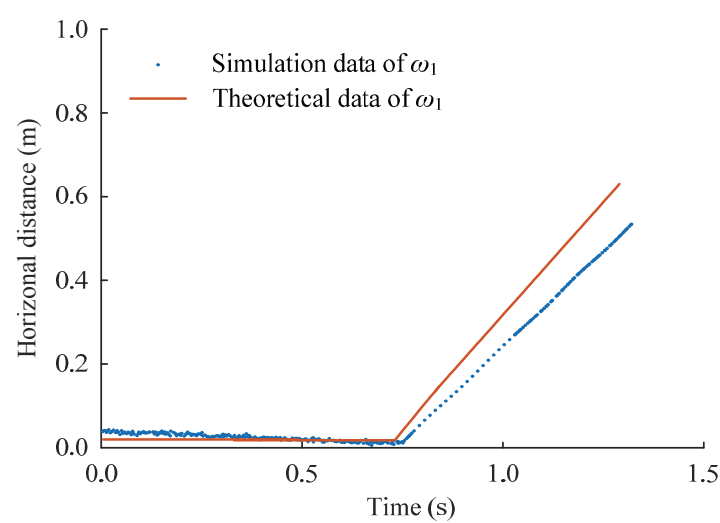

(a)

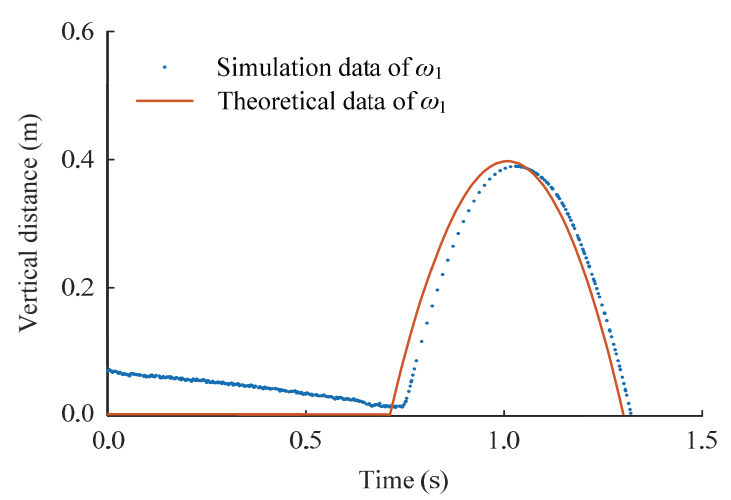

(b)

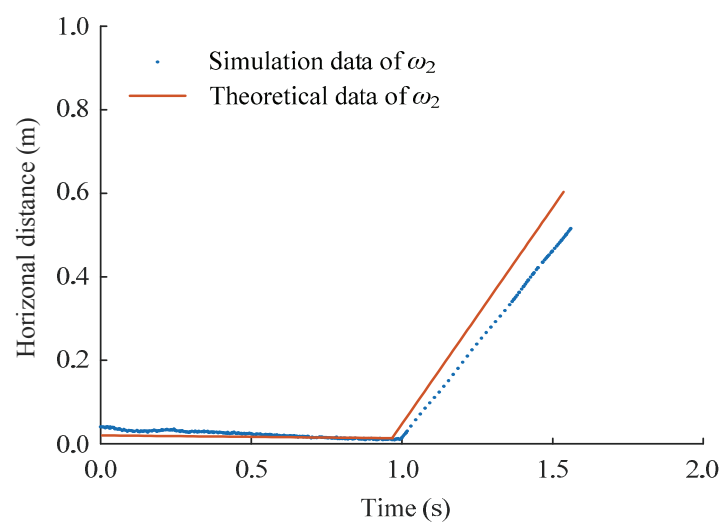

(c)

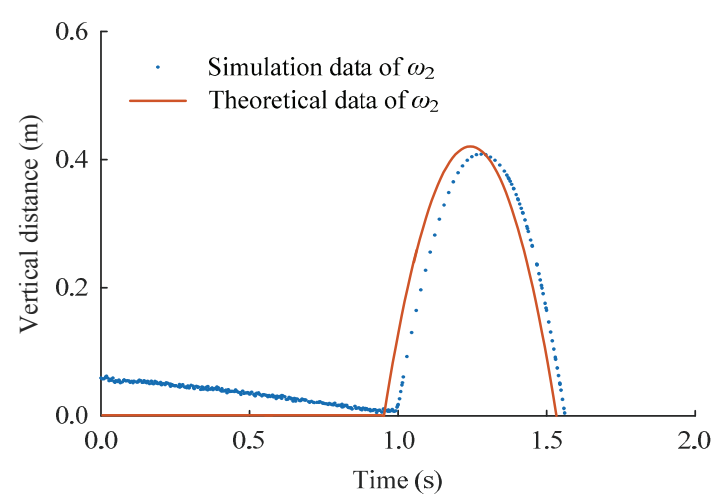

(d)

Fig. 11 Hopping height and forward distance of theory and simulation results in the air-flying stage. (a) Forward distance in theory calculation and simulation with the actuating speed $\omega_{1} ;$ (b) hopping height in theory calculation and simulation with the actuating speed $\omega_{1} ;(\mathrm{c})$ forward distance in theory calculation and simulation with the actuating speed $\omega_{2} ;$ (d) hopping height in theory calculation and simulation with the actuating speed $\omega_{2}$.

occurs and affects the flying trajectory. Thus, the robot hopping distance in the horizontal direction is less than the theoretically calculated value.
The experiment was carried out using the motor at different drive speeds although the drive speed was the same as that in the simulation. The animations of two 
simulated hopping processes and two experimental ones are presented in Fig. 12. The hopping height and forward distance of the robot in the first simulation are $0.38 \mathrm{~m}$ and $0.53 \mathrm{~m}$, and those are $0.42 \mathrm{~m}$ and $0.51 \mathrm{~m}$ in the second simulation. The experiment results show that the robot can hop about $0.338 \mathrm{~m}$ high and $0.45 \mathrm{~m}$ far with the actuating speed $\omega_{1}$. The height and the forward distance of the hopping with the actuating speed $\omega_{2}$ are $0.368 \mathrm{~m}$ and $0.329 \mathrm{~m}$ respectively. These results are like the simulation results although there are few differences that are caused by machining and assembly errors exist in the real robot. Like simulation, the rolling phenomenon in the hopping also happens in the experiment. Some kinetic energy is used for rolling motion in the air-flying stage. Thus, the hopping data has some difference with that in the theoretical calculation. To sum up, the dynamics of the multi-motion states in a hopping cycle of the robot is verified by theoretical calculation, simulations, and experiments.

\section{Discussion}

The objective of this study is to design a simple, small, compact, and independent hopping robot with the inspiration of jumping mechanism of click beetles and analyse the dynamics of the robot during the three stages in the jumping process to finally form the dynamic synthesis theory.

We developed a single-legged robot inspired by the jumping mechanism of click beetles. The click beetle applies the hinge part combined with the body to jump. It is simple and suitable as an inspiration to imitate and design a new hopping robot. The height and length of the robot is $85 \mathrm{~mm}$ and $60 \mathrm{~mm}$, and the mass is about $49 \mathrm{~g}$. It has good hopping performance which was tested with simulation and experiment. Its hopping height is about $4-4.3$ times of its body height. This hopping result is superior to galago's one jump height without wall jump that is about 3.2 times of its body height (exactly the jumping height galago is about $80 \mathrm{~cm}$ with about $25 \mathrm{~cm}$ of the body height ${ }^{[15]}$ ). Thus, it is simple, small, compact, and independent for rescue missions that require to enter enclosed spaces through cracks and narrow channels.

The hopping process of the jumping robots mostly includes three stages: pre-hopping, take-off, and air-flying stage. The backward stages are dependent on the forward one. The pre-hopping stage supplies the hopping energy for the take-off and air-flying stage, and the final velocity of the robot in the take-off stage determines the hopping distance of the air-flying stage. Thus, the dynamic analysis containing the three stages is very necessary for understanding the hopping and improving the hopping performance.

We applied the decomposition method to complete the hopping dynamic model construction. First, the hopping dynamics of the three stages were analyzed respectively. Then, forward and backward stage was related by the dependent parameter. To be specific, the torque obtained at the end of pre-hopping stage was used as the driving torque of the take-off stage, and the velocity of the robot in the end of the take-off stage was applied to calculate the air-flying trajectory. Finally, the three stage dynamics of the hopping were synthesized. In dynamic analysis of hopping, Newton-Euler dynamic analysis method and lumped-mass method were mainly employed. In the existing dynamic analysis of the hopping robots, such as linkage type and bionic types, only one or two stages of the hopping process were analyzed. In this study, we studied the dynamic of the three hopping stages and proposed the synthesized dynamic theory to describe the whole hopping process of the hopping robots.

The hopping synthesized dynamic theory of hopping robots was verified by simulations and experiments. In the pre-hopping stage, results of the joint torque were almost same between the theoretical calculation and the simulation. But this stage in simulation ended a little later than that in the theoretical calculation. It is mainly caused by the side clearance of the gear in the simulation which was not considered in the theoretical calculation. In the take-off stage, the angular velocity in the simulation was larger than that in the theoretical calculation. It is because lumped-mass method was used in the theoretical calculation which caused this difference especially in the high velocity situation. In the air-flying stage, the hopping height and forward distance of the robot in theoretical calculations, simulations, and experiments were respectively $(0.40 \mathrm{~m}, 0.62 \mathrm{~m}),(0.43 \mathrm{~m}$, $0.61 \mathrm{~m}) ;(0.38 \mathrm{~m}, 0.53 \mathrm{~m}),(0.42 \mathrm{~m}, 0.51 \mathrm{~m})$; and $(0.34 \mathrm{~m}$, $0.45 \mathrm{~m}),(0.37 \mathrm{~m}, 0.33 \mathrm{~m})$. Most of them were consistent except the data of forward distance. In detail, the 


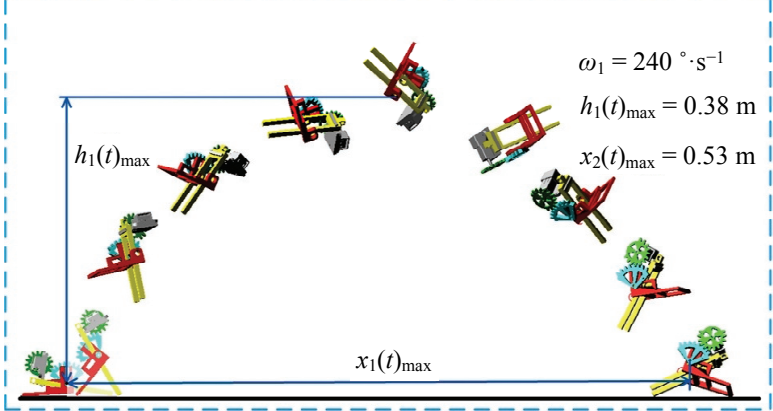

(a)

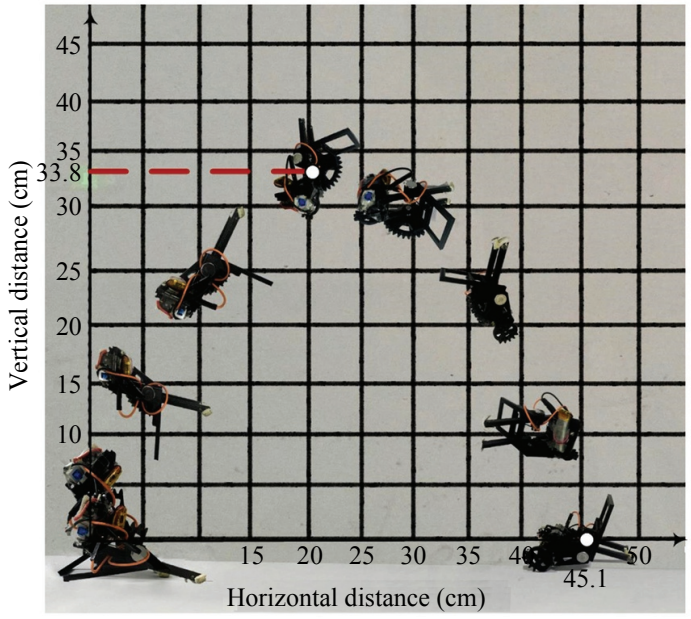

(c)

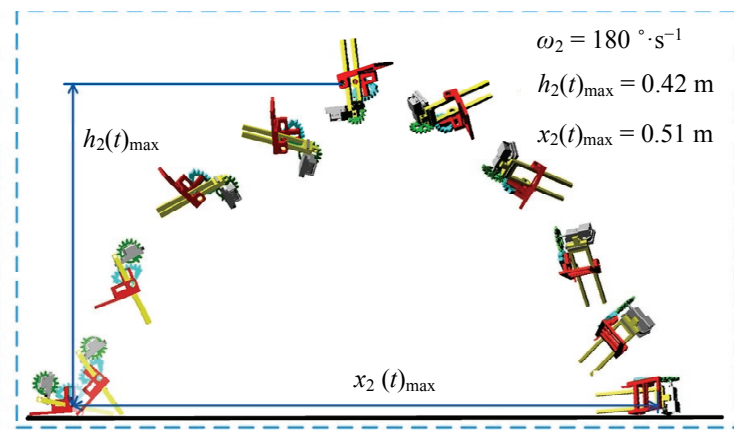

(b)

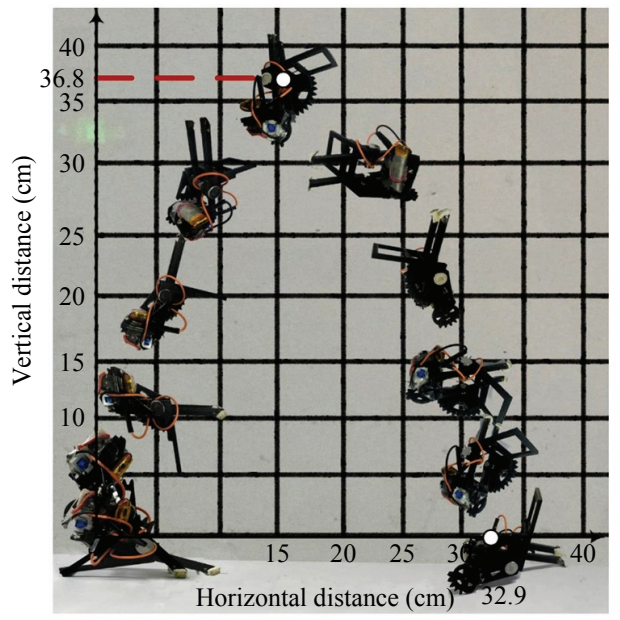

(d)

Fig. 12 Videos of the hopping process in simulations and experiments. (a) Hopping simulation with the actuating speed $\omega_{1}$, and the hopping height and forward distance are $0.38 \mathrm{~m}$ and $0.53 \mathrm{~m}$; (b) hopping simulation with the actuating speed $\omega_{2}$, and the hopping height and forward distance are $0.42 \mathrm{~m}$ and $0.51 \mathrm{~m}$; (c) hopping experiment with the actuating speed $\omega_{1}$, and the hopping height and forward distance are $0.34 \mathrm{~m}$ and $0.45 \mathrm{~m}$; (d) hopping experiment with the actuating speed $\omega_{2}$, and the hopping height and forward distance are $0.37 \mathrm{~m}$ and $0.33 \mathrm{~m}$.

forward distance of the robot in theoretical calculations had about $0.1 \mathrm{~m}$ to $0.3 \mathrm{~m}$ difference with those in simulations, and experiments. This is caused by the simplification that the rolling motion of the robot in the air-flying stage was unconsidered in the theoretical calculation, which have a great influence on the forward distance result. This will be improved in the future study.

The simulation and experiment results demonstrate that the synthesized dynamic theory of hopping robots is correct and valid. This proposed synthesized dynamics improves the hopping dynamic theory in the hopping robot study and can be used as the basic method for understanding the hopping and improving the hopping performance. It will promote the development of dynamic analysis and calculation of hopping robots.

\section{Conclusion}

Inspired by the jumping mechanism of click beetles, a simple, small, and reliable hopping robot with a single leg was developed through the meshing and disengagement of the gear set combined with torsional spring for storing and releasing the energy. The robot has good hopping performance that the hopping height is about 4 -4.3 times of its body height. The robot is simple, small, compact, independent and has good hopping ability that it gives a new application for rescue missions that require to enter enclosed spaces through cracks and narrow channels. Three motion states exhibit different dynamic characteristics in the hopping process of the robot. The dynamics of the multi-motion states during a hopping period was analyzed and the dynamic synthesis model was established based on these motion states. The robot was firstly simulated by ADAMS, including the multi-motion states during the hopping. A robot platform was then used in the experiments to verify the dynamic model of the multi-motion states further. The 
theoretical calculations, simulation and experimental results clearly showed that the proposed approach was highly consistent although some small differences were observed in the dynamic curves of the hopping. The dynamic synthesis of multi-motion states in a hopping cycle of the robot has formed the theoretical foundation in this study for the potential comprehensive analysis of the jumping process of leg-type hopping robot. Our future works will focus on the optimization, reliability, and service life of the robot structures, self-righting control after take-off, and the fall handling.

\section{Acknowledgment}

This work was financially supported by National Natural Science Foundation of China (Nos. 51875528 and 41506116), Zhejiang Provincial Natural Science Foundation of China (No. LY20E050018), China Postdoctoral Science Foundation (No. 2016M591991), and Science Foundation of Zhejiang Sci-Tech University (ZSTU) (No. 17022183-Y).

Open Access This article is licensed under a Creative Commons Attribution 4.0 International License, which permits use, sharing, adaptation, distribution and reproduction in any medium or format, as long as you give appropriate credit to the original author(s) and the source, provide a link to the Creative Commons licence, and indicate if changes were made.

The images or other third party material in this article are included in the article's Creative Commons licence, unless indicated otherwise in a credit line to the material. If material is not included in the article's Creative Commons licence and your intended use is not permitted by statutory regulation or exceeds the permitted use, you will need to obtain permission directly from the copyright holder.

To view a copy of this licence, visit http://creativecommons.org/licenses/by/4.0/.

\section{References}

[1] Johnson A M, Koditschek D E. Toward a vocabulary of legged leaping. Proceedings of the IEEE International Conference on Robotics and Automation, Karlsruhe, Germany, 2013, 2568-2575.

[2] Desbiens A L, Pope M T, Christensen D L, Hawkes E W, Cutkosky M R. Design principles for efficient, repeated jumpgliding. Bioinspiration \& Biomimetics, 2014, 9, 025009.

[3] Fiorini P, Burdick J W. The development of hopping capabilities for small robots. Autonomous Robots, 2003, 14, 239-254.

[4] Chen D S, Zhang Z Q, Chen K W. Dynamic model and performance analysis of landing buffer for bionic locust mechanism. Acta Mechanica Sinica, 2016, 32, 551-565.

[5] Zhang J, Song G M, Qiao G F, Meng T H, Sun H T. An indoor security system with a jumping robot as the surveillance terminal. IEEE Transactions on Consumer Electronics, 2011, 57, 1774-1781.

[6] Song S G, Wang C J. Landing stability analysis of the lunar lander based on Monte Carlo approach. Journal of Beijing University of Aeronautics and Astronautics, 2013, 39, 1192-1196. (in Chinese)

[7] Lin Q, Nie H, Xu L, Chen J B. Analysis on key influence factors of soft-landing stability in lunar lander. Journal of Nanjing University of Aeronautics \& Astronautics, 2012, 44, 152-158. (in Chinese)

[8] Zhang Z Q, Chen D S, Chen K W, Chen H L. Analysis and comparison of two jumping leg models for bioinspired locust robot. Journal of Bionic Engineering, 2016, 13, 558-571.

[9] Chen D S, Zhang Z Q, Chen K W. Legs attitudes determination for bionic locust robot based on landing buffering performance. Mechanism and Machine Theory, 2016, 99, 117-139.

[10] Zhang J, Song G M, Li Y Y, Qiao G F, Song A G, Wang A M. A bio-inspired jumping robot: Modeling, simulation, design, and experimental results. Mechatronics, 2013, 23, 1123-1140.

[11] Vu H Q, Yu X X, Iida F, Pfeifer R. Improving energy efficiency of hopping locomotion by using a variable stiffness actuator. IEEE/ASME Transactions on Mechatronics, 2016, 21, 472-486.

[12] Zhang Y H, Zhang L F, Wang W, Li Y M, Zhang Q W. Design and implementation of a two-wheel and hopping robot with a linkage mechanism. IEEE Access, 2018, 6, 42422-42430.

[13] Kenneally G, De A, Koditschek D E. Design principles for a family of direct-drive legged robots. IEEE Robotics and Automation Letters, 2016, 1, 900-907.

[14] Jung G P, Casarez C S, Jung S P, Fearing R S, Cho K J. An integrated jumping-crawling robot using height-adjustable jumping module. IEEE International Conference on Robotics and Automation, Stockholm, Sweden, 2016, 
4680-4685.

[15] Haldane D W, Plecnik M M, Yim J K, Fearing R S. Robotic vertical jumping agility via series-elastic power modulation. Science Robotics, 2016, 1, eaag2048.

[16] Kovač M, Schlegel M, Zufferey J, Floreano D. A miniature jumping robot with self-recovery capabilities. IEEE/RSJ International Conference on Intelligent Robots and Systems, St. Louis, MO, USA, 2009, 583-588.

[17] Plecnik M M, Haldane D W, Yim J K, Fearing R S. Design exploration and kinematic tuning of a power modulating jumping monopod. Journal of Mechanisms and Robotics, 2017, 9, 011009.

[18] Haldane D W, Plecnik M, Yim J K, Fearing R S. A power modulating leg mechanism for monopedal hopping. IEEE/RSJ International Conference on Intelligent Robots and Systems, Daejeon, South Korea, 2016, 4757-4764.

[19] Graichen K, Hentzelt S, Hildebrandt A, Kärcher N, Gaißert N, Knubben E. Control design for a bionic kangaroo. Control Engineering Practice, 2015, 42, 106-117.

[20] Scarfogliero U, Stefanini C, Dario P. Design and development of the long-jumping "grillo" mini robot. Proceedings of IEEE International Conference on Robotics and Automation, Roma, Italy, 2007, 467-472.

[21] Scarfogliero U, Stefanini C, Dario P. The use of compliant joints and elastic energy storage in bio-inspired legged robots. Mechanism and Machine Theory, 2009, 44, 580-590.

[22] Beck A, Zaitsev V, Hanan U B, Kosa G, Ayali A, Weiss A. Jump stabilization and landing control by wing-spreading of a locust-inspired jumper. Bioinspiration \& Biomimetics, 2017, 12, 066006.

[23] Zaitsev V, Gvirsman O, Hanan U B, Weiss A, Ayali A, Kosa G. A locust-inspired miniature jumping robot. Bioinspiration \& Biomimetics, 2015, 10, 066012.

[24] Nguyen Q, Park H C. Design and demonstration of a locust-like jumping mechanism for small-scale robots. Journal of Bionic Engineering, 2012, 9, 271-281.

[25] Wang M, Zang X Z, Fan J Z, Zhao J. Biological jumping mechanism analysis and modeling for frog robot. Journal of Bionic Engineering, 2008, 5, 181-188.

[26] Reddy N S, Ray R, Shome S N. Modeling and simulation of a jumping frog robot. IEEE International Conference on Mechatronics and Automation, Beijing, China, 2011, 1264-1268.

[27] De Cubber G, Serrano D, Berns K, Chintamani K, Sabino R, Ourevitch S, Doroftei D, Armbrust C, Flamma T, Baudoin Y. Search and rescue robots developed by the european icarus project. 7th International Workshop on Robotics for Risky Environments, Saint-Petersburg, Russia, 2013.

[28] Ribak G, Reingold S, Weihs D. The effect of natural substrates on jump height in click-beetles. Functional Ecology, 2012, 26, 493-499.

[29] Ribak G, Mordechay O, Weihs D. Why are there no long distance jumpers among click-beetles (Elateridae)? Bioinspiration \& Biomimetics, 2013, 8, 036004.

[30] Bolmin O, Duan C F, Urrutia L, Abdulla A M, Hazel A M, Alleyne M, Dunn A C, Wissa A. Pop! Observing and modeling the legless self-righting jumping mechanism of click beetles. Conference on Biomimetic and Biohybrid Systems, Stanford, CA, USA, 2017, 35-47.

[31] Yang X F. Trigger of click beetles in Dishuiyan. https://mp.weixin.qq.com/s/kDms2jTgA6Qq9jMJmuyuWg, 2018. (in Chinese)

[32] Chen D S, Chen K W, Zhang Z Q, Zhang B G. Mechanism of locust air posture adjustment. Journal of Bionic Engineering, 2015, 12, 418-431.

[33] Zhao J G, Xi N, Cintrón F J, Mutka M W, Xiao L. A single motor actuated miniature steerable jumping robot. IEEE/RSJ International Conference on Intelligent Robots and Systems, Vilamoura, Portugal, 2012, 4274-4275.

[34] Zhang Z Q, Chen D S, Chen K W, Chen H L. Analysis and comparison of two jumping leg models for bioinspired locust robot. Journal of Bionic Engineering, 2016, 13, 558-571.

[35] Li F, Liu W T, Fu X, Bonsignori G, Scarfogliero U, Stefanini C, Dario P. Jumping like an insect: Design and dynamic optimization of a jumping mini robot based on bio-mimetic inspiration. Mechatronics, 2012, 22, 167-176.

[36] Kumar G, Pathak P M. Dynamic modelling \& simulation of a four legged jumping robot with compliant legs. Robotics and Autonomous Systems, 2013, 61, 221-228.

[37] Evans M E G. The jump of the click beetle (Coleoptera, Elateridae) - A preliminary study. Journal of Zoology, 1972, 167, 319-336.

[38] Ribak G, Weihs D. Jumping without using legs: The jump of the click-beetles (Elateridae) is morphologically constrained. PLOS ONE, 2011, 6, e20871.

[39] Bolmin O, Wei L H, Hazel A M, Dunn A C, Wissa A, Alleyne M. Latching of the click beetle (Coleoptera: Elateridae) thoracic hinge enabled by the morphology and mechanics of conformal structures. Journal of Experimental Biology, 2019, 222, jeb196683. 


\section{Appendix}

\section{Parameters of the single-legged hopping robot}

Parameters of the single-legged hopping robot are described in Table A1.

Table A1 Parameters of the robot

\begin{tabular}{ccc}
\hline Symbol & Unit & Value \\
\hline Length & $\mathrm{m}$ & 0.060 \\
Width & $\mathrm{m}$ & 0.041 \\
Height & $\mathrm{m}$ & 0.085 \\
$l_{1}$ & $\mathrm{~m}$ & 0.045 \\
$l_{2}$ & $\mathrm{~m}$ & 0.070 \\
$\mathrm{~A}$ & $\mathrm{~m}$ & 0.015 \\
$\mathrm{~B}$ & $\mathrm{~m}$ & 0.025 \\
$m_{1}$ & $\mathrm{~kg}$ & 0.012 \\
$m_{2}$ & $\mathrm{~kg}$ & 0.014 \\
$m_{3}$ & $\mathrm{~kg}$ & 0.023 \\
$J_{1}$ & $\mathrm{~kg} \cdot \mathrm{m}^{2}$ & $4.23 \mathrm{e}^{-4}$ \\
$J_{2}$ & $\mathrm{~kg} \cdot \mathrm{m}^{2}$ & $3.71 \mathrm{e}^{-5}$ \\
$\theta_{21}$ & degree & 80 \\
$\theta_{22}$ & degree & 10 \\
$\mathrm{~K}$ & & 52.000 \\
\hline
\end{tabular}

\section{Kinematics of the single-legged hopping robot}

Fig. A1 shows the established coordinate system. Three frames $\{0\},\{1\}$, and $\{2\}$ are defined. The D-H parameters are shown in Table A2, and the symbols are depicted in Fig. A1.

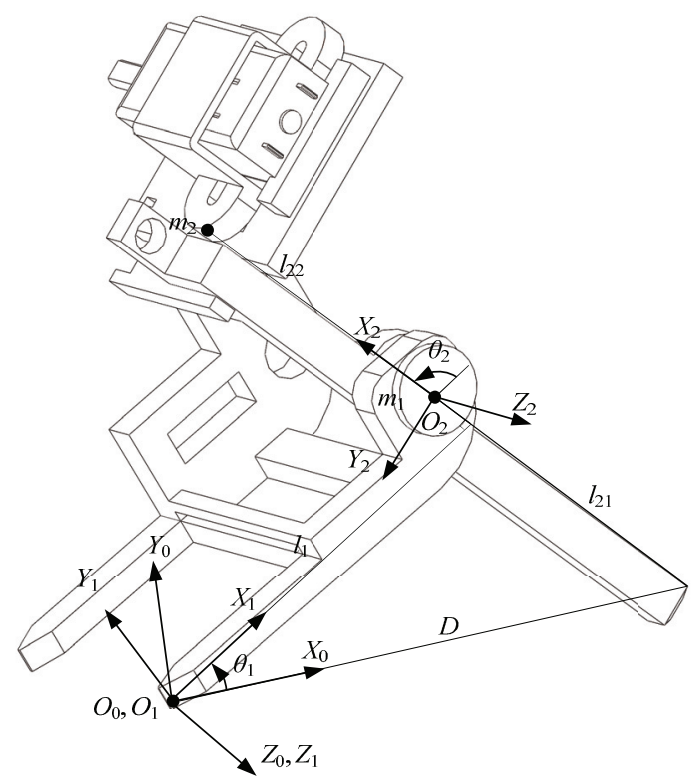

Fig. A1 Coordinate systems defined in the single-legged hopping robot model. Three coordinate systems are built and symbols are described. 
Table A2 D-H parameters

\begin{tabular}{ccccc}
\hline$i$ & $\alpha_{i-1}$ & $a_{i-1}$ & $d_{i}$ & $\theta_{i}$ \\
\hline 1 & 0 & 0 & 0 & $\theta_{1}$ \\
2 & 0 & $l_{1}$ & 0 & $\theta_{2}$ \\
\hline
\end{tabular}

The relationship between the coordinate systems is given by the following:

$$
\begin{aligned}
& { }_{0}^{1} \boldsymbol{R}=\left(\begin{array}{ccc}
\cos \theta_{1} & \sin \theta_{1} & 0 \\
-\sin \theta_{1} & \cos \theta_{1} & 0 \\
0 & 0 & 1
\end{array}\right), \\
& { }_{1}^{2} \boldsymbol{R}=\left(\begin{array}{ccc}
\cos \theta_{2} & \sin \theta_{2} & 0 \\
-\sin \theta_{2} & \cos \theta_{2} & 0 \\
0 & 0 & 1
\end{array}\right),
\end{aligned}
$$

Link centroid positions are:

$$
\begin{aligned}
& { }^{1} \boldsymbol{P}_{c_{1}}=l_{1} \hat{\boldsymbol{X}}_{1}, \\
& { }^{2} \boldsymbol{P}_{\mathrm{c}_{2}}=-l_{22} \hat{\boldsymbol{X}}_{2} .
\end{aligned}
$$

Assume that the masses of the main leg and lower leg are $m_{1}, m_{2}$ and they are concentrated.

The link inertia tensors are zero matrices as follows:

$$
\begin{aligned}
{ }^{c 1} \boldsymbol{I}_{1} & =0, \\
{ }^{c 2} \boldsymbol{I}_{2} & =0 .
\end{aligned}
$$

When the robot is fixed in the hind foot during the movement, we obtain the following:

$$
\begin{gathered}
\omega_{0}=0, \\
\dot{\omega}_{0}=0 .
\end{gathered}
$$

As shown in Fig. A1, the geometrical relationship between the two legs is obtained by:

$$
\frac{D}{\sin \theta_{2}}=\frac{l_{21}}{\sin \theta_{1}},
$$

where $D$ is the distance between the main leg and the lower leg,

$$
\begin{aligned}
& D=\sqrt{l_{21}^{2}+l_{1}^{2}+2 l_{21} l_{1} \cos \theta_{2}}, \\
& \theta_{1}=\arcsin \left(\frac{l_{21}}{\sqrt{l_{21}^{2}+l_{1}^{2}+2 l_{21} l_{1} \cos \theta_{2}}} \sin \theta_{2}\right), \\
& \theta_{1}=90^{\circ}-0.5 \theta_{2} .
\end{aligned}
$$

\section{Velocity and acceleration analysis of the robot in the pre-hopping and take-off stage}

The outward iteration method for the lower leg is solved, and its angular velocity in frame $\{1\}$ is as: 


$$
{ }^{1} \omega_{1}={ }_{0}^{1} R^{0} \omega_{0}+\dot{\theta}_{1} \widehat{Z}_{1}=\left[\begin{array}{c}
0 \\
0 \\
\dot{\theta}_{1}
\end{array}\right]
$$

The angular acceleration of the lower leg in frame $\{1\}$ is obtained below:

$$
{ }^{1} \dot{\boldsymbol{\omega}}_{1}={ }_{0}^{1} \boldsymbol{R}{ }^{0} \boldsymbol{\omega}_{0}+{ }_{0}^{1} \boldsymbol{R}^{0} \boldsymbol{\omega}_{0} \times \dot{\theta}_{1}^{1} \widehat{\boldsymbol{Z}}_{1}+\ddot{\theta}_{1} \widehat{\boldsymbol{Z}}_{1}=\left[\begin{array}{c}
0 \\
0 \\
\ddot{\theta}_{1}
\end{array}\right] .
$$

The acceleration of the centre of the lower leg in frame $\{0\}$ is as:

$$
{ }^{0} \dot{\boldsymbol{v}}_{0}=g \cdot \hat{\boldsymbol{Y}}_{0} .
$$

The acceleration of the centre of the lower leg in frame $\{1\}$ is obtained as:

$$
\begin{gathered}
{ }^{1} \dot{\boldsymbol{v}}_{1}={ }_{0}^{1} \boldsymbol{R}\left({ }^{0} \dot{\boldsymbol{\omega}}_{0} \times{ }^{0} \boldsymbol{P}_{1}+{ }^{0} \boldsymbol{\omega}_{0} \times\left({ }^{0} \boldsymbol{\omega}_{0} \times{ }^{0} \boldsymbol{P}_{1}\right)+{ }^{0} \dot{\boldsymbol{v}}_{0}\right)=\left[\begin{array}{c}
g \sin \theta_{1} \\
g \cos \theta_{1} \\
0
\end{array}\right], \\
{ }^{1} \dot{\boldsymbol{v}}_{c 1}={ }^{1} \dot{\boldsymbol{\omega}}_{1} \times{ }^{1} \boldsymbol{P}_{C_{1}}+{ }^{1} \boldsymbol{\omega}_{1} \times\left({ }^{1} \boldsymbol{\omega}_{1} \times{ }^{1} \boldsymbol{P}_{C_{1}}\right)+{ }^{1} \dot{\boldsymbol{v}}_{1}=\left[\begin{array}{c}
-l_{1} \dot{\theta}_{1}^{2}+g \sin \theta_{1} \\
l_{1} \ddot{\theta}_{1}+g \cos \theta_{1} \\
0
\end{array}\right] .
\end{gathered}
$$

The outer iterative method for connecting rod 2 is solved as:

$$
\begin{gathered}
{ }^{2} \boldsymbol{\omega}_{2}=\left[\begin{array}{c}
0 \\
0 \\
\dot{\theta}_{1}+\dot{\theta}_{2}
\end{array}\right], \\
{ }^{2} \dot{\boldsymbol{\omega}}_{2}=\left[\begin{array}{c}
0 \\
0 \\
\ddot{\theta}_{1}+\ddot{\theta}_{2}
\end{array}\right] .
\end{gathered}
$$

The angular speed of the main leg is obtained below:

$$
{ }^{2} \dot{\boldsymbol{v}}_{2}=\left(\begin{array}{ccc}
\cos \theta_{2} & \sin \theta_{2} & 0 \\
-\sin \theta_{2} & \cos \theta_{2} & 0 \\
0 & 0 & 1
\end{array}\right)\left[\begin{array}{c}
-l_{1} \dot{\theta}_{1}^{2}+g \sin \theta_{1} \\
l_{1} \ddot{\theta}_{1}+g \cos \theta_{1} \\
0
\end{array}\right]=\left[\begin{array}{c}
-l_{1} \dot{\theta}_{1}^{2} \cos \theta_{2}+l_{1} \ddot{\theta}_{1} \sin \theta_{2}+g \sin \left(\theta_{1}+\theta_{2}\right) \\
l_{1} \dot{\theta}_{1}^{2} \sin \theta_{2}+l_{1} \ddot{\theta}_{1} \cos \theta_{2}+g \cos \left(\theta_{1}+\theta_{2}\right) \\
0
\end{array}\right] .
$$

The main leg acceleration at the centre of mass is obtained here:

$$
{ }^{2} \dot{\boldsymbol{v}}_{c 2}={ }^{2} \dot{\boldsymbol{v}}_{2}+{ }^{2} \dot{\boldsymbol{\omega}}_{2} \times{ }^{2} \boldsymbol{P}_{\mathrm{c} 2}+{ }^{2} \boldsymbol{\omega}_{2} \times\left({ }^{2} \boldsymbol{\omega}_{2} \times{ }^{2} \boldsymbol{P}_{\mathrm{c} 2}\right)=\left[\begin{array}{c}
-l_{22}\left(\dot{\theta}_{1}+\dot{\theta}_{2}\right)^{2}-l_{1} \dot{\theta}_{1}^{2} \cos \theta_{2}-l_{1} \ddot{\theta}_{1} \sin \theta_{2}+g \sin \left(\theta_{1}+\theta_{2}\right) \\
l_{22}\left(\ddot{\theta}_{1}+\ddot{\theta}_{2}\right)+l_{1} \dot{\theta}_{1}^{2} \sin \theta_{2}+l_{1} \ddot{\theta}_{1} \cos \theta_{2}+g \cos \left(\theta_{1}+\theta_{2}\right) \\
0
\end{array}\right] .
$$

